\title{
EL ENVEJECIMIENTO RURAL COMO FACTOR NEGATIVO EN LA PRODUCTIVIDAD AGRÍCOLA EN EL MAGREB
}

\author{
Nieva Machín ${ }^{1}$ y Eric Pardo² \\ Universidad Complutense de Madrid / UNISCI
}

\begin{abstract}
Resumen:
Este artículo se centra en la consideración de dos factores a los que se enfrenta en campo magrebí en Marruecos, Argelia y Túnez y que son tanto el envejecimiento de su población rural como las deficiencias estructurales que inciden en el desinterés de la población más joven por permanecer en el medio rural. En primer lugar se revisan las tendencias actuales en lo que a envejecimiento se refiere, para así determinar que el envejecimiento del agro magrebí se debe principalmente al fenómeno de emigración hacia las ciudades. Seguidamente se explican las deficiencias estructurales existentes, centradas principalmente en el escaso apoyo institucional, la baja capacitación de los agricultores, las deficiencias en infraestructuras, sin olvidar claro está, las menciones al cambio climático y al proceso de desertización. De esta explicación se deduce que ambos factores se retroalimentan, siendo cada vez más difícil mejorar la situación a medida que se profundicen ambos fenómenos, pues las deficiencias estructurales privan de oportunidades a los jóvenes agricultores y dejan a una población envejecida menos capacitada para introducir mejoras en la agricultura. La víctima de este círculo vicioso será la productividad y la seguridad alimentaria.
\end{abstract}

Palabras clave: Envejecimiento, demografía, emigración, productividad, seguridad alimentaria.

Title in English: "Rural Ageing as a Negative Factor in the Maghreb and its Effects on Agrarian Productivity".

\begin{abstract}
:
This article deals with two factors affecting agriculture in three Maghreb countries, Morocco, Algeria and Tunisia: rural ageing and structural deficiencies in the agriculture sector, which will arguably worsen in the future and which have a strong influence on young populations for migrating to the urban milieu in search for better opportunities. We will first review current tendencies in the process of ageing in these countries to conclude that most of the rural ageing finds its roots in migration into the cities. After that, we will deal with the structural deficiencies which are mainly related to lack of institutional support, to the low level of skill in current farmers and to the underdevelopment of infrastructures, not forgetting to mention climate change and the related process of desertification. We will conclude that these two factors feedback each other, being the fact that whenever both phenomena deepen, it will be more difficult to favourably change the situation, as structural deficiencies reduce opportunities for young potential farmers who will then immigrate and leave behind an aged population unfit for introducing improvements in agriculture, and reproducing then the process of emigration. The consequence of this vicious circle will be both reduced productivity and food security.
\end{abstract}

Keywords: Ageing, Demographics, Emigration, Productivity, Food Security.

Copyright $\odot$ UNISCI, 2013.

Las opiniones expresadas en estos artículos son propias de sus autores, y no reflejan necesariamente la opinión de UNISCI. The views expressed in these articles are those of the authors, and do not necessarily reflect the views of UNISCI.

\footnotetext{
${ }^{1}$ Nieva Machín Osés es investigadora junior de UNISCI y miembro del Foro Hispano-Argelino.

${ }^{2}$ Eric Pardo Sauvageot es estudiante de doctorado en el Departamento de Estudios Internacionales de la Facultad de Ciencias Políticas y Sociología de la Universidad Complutense, es investigador junior de UNISCI, miembro del Foro Hispano-Argelino y está disfrutando de una estancia de investigación en el Centro Aleksanteri de Estudios Rusos y de Europa Oriental, Helsinki, Finlandia, de enero a abril del 2013 becado por el CIMO, Ministerio de Educación de Finlandia.
}

http://dx.doi.org/10.5209/rev_UNIS.2013.n31.4474ロ 


\section{Introducción}

En este capítulo vamos a comentar la existencia de un proceso de envejecimiento del campo de los tres países del Magreb que van a entrar en nuestro análisis, Marruecos, Argelia y Túnez, y ligarlo por tanto con el problema de sostenibilidad agrícola explicando también los diversos factores ya existentes y que minan de por sí el futuro de la producción agrícola de estos países. La hipótesis que queremos manejar por tanto es que estos países se enfrentan a un panorama crítico en lo que a sostenibilidad agrícola se refiere, ya que esta población envejecida no podrá sostener una agricultura modernizada que supere los problemas estructurales existentes.

Lo peor de todo es que este fenómeno se da además justo cuando, como tenemos ocasión de ver en el capítulo de los profesores David Cantalapiedra y Rubén Herrero, los efectos del cambio climático en el área geográfica de estos tres países van a hacerse notar cada vez más, siendo la consecuencia directa de ello la desertización provocada por las caídas en la pluviosidad en esta región a que los diversos modelos científicos manejados en estos momentos apuntan; ello inevitablemente habrá de afectar al rendimiento agrario, con zonas de rendimiento decreciente y otras totalmente inaptas para la agricultura.

\subsection{Predicciones en demografía}

El envejecimiento por razones demográficas es ya una realidad, como puede deducirse de las diversas predicciones que se manejan. Este envejecimiento, es en todo caso por el momento bastante relativo, pues en el caso del Magreb, las predicciones aún muestran tendencias de crecimiento poblacional que en Europa han quedado ya atrás: la progresión de la población en todo el Oriente Próximo, región en la que se engloba el Magreb y que comparte muchas características con esta última, será espectacular en comparación con el panorama que encontramos en Europa: del 2010 al 2050, la población aumentará en nada más ni nada menos que en un 62\%; en comparación, haciendo caso de los datos de Eurostat, Europa también crecerá, pero lo hará en una proporción sensiblemente menor, pasando de 501 millones de habitantes en 2010 a 517 millones cincuenta años más tarde en el 2060.

En el caso del Magreb, tenemos el ejemplo de Marruecos donde la población debería pasar de 30 millones en el año 2004 a 38 millones en el $2030^{3}$, Argelia donde de 37 millones en 2012 se prevé una duplicación de la población hasta los 70 millones en $2050^{4}$ o Túnez, que con una población de alrededor de 10 millones en 2010, podrían alcanzar los 13 millones en 2034 . Según datos del Banco Mundial, Marruecos pasó de 31 millones de habitantes en el 2007 a 32 millones cuatro años más tarde en el 2011, siendo la progresión en este mismo período de casi 34 millones a cerca de 36 millones para Argelia y de 10,22 millones a 10,67 millones para Túnez ${ }^{6}$. Las predicciones que maneja el portal especializado Population Pyramid para 2050 son las siguientes: si Argelia contaba con 35 millones y medio en el $2010^{7}$, en el 2050 se proyecta una población notablemente superior con 46 millones y medio ${ }^{8}$,

Géopopulation: "Démographie Maroc: vieillissement de la population jusqu'en 2030", en http://www.geopopulation.com/20090117/demographie-maroc-vieillissement-de-la-population-jusquen-2030/.

${ }^{4}$ Bladi, Oueld: "La démographie algérienne en chiffres", Blog Algérie, 13 junio 2012, en http://blogalgerie.com/la-demographie-algerienne-en-chiffres.

${ }^{5}$ Géopopulation: "Démographie Tunisie: la population devrait augmenter et vieillir - Projection 2034", 03 noviembre 2009, en http://www.geopopulation.com/20091114/demographie-tunisie-la-population-devraitaugmenter-et-vieillir-projection-2034/.

${ }^{6}$ El Banco Mundial: " Population, total", en http://data.worldbank.org/indicator/SP.POP.TOTL.

${ }^{7}$ Population Pyramid: "Algeria 2010", en http://populationpyramid.net/Algeria/2010/.

${ }^{8}$ Population Pyramid: "Algeria 2050", en http://populationpyramid.net/Algeria/2050/. 
datos que precisamente vienen corroborados por las estimaciones medias realizadas por la $\mathrm{ONU}^{9}$; en el caso de Marruecos y Túnez, con las mismas fechas de referencia, las predicciones son de un aumento de población, desde cerca de 32 millones $^{10}$ a 39 millones para el primero $^{11}$, y de 10 millones y medio ${ }^{12}$ a 12 millones y medio ${ }^{13}$ para el segundo. En el caso de estos dos países, las predicciones igualmente coinciden con la variable media de la oficina de población de la ONU ${ }^{14}$.

\subsection{Características de la transición demográfica}

Este aumento demográfico es relativo, pues el Magreb se encuentra ya en una fase de transición, que además está teniendo un carácter muy drástico: si en 1970 la tasa de fecundidad era de unos 7 hijos por mujer ${ }^{15}$, en 2010, se había ya alcanzado la tasa más propia de países desarrollados, de 2 hijos por mujer ${ }^{16}$; si el Magreb era hace varias décadas la región del mundo con la mayor explosión demográfica, ahora precisamente se encuentra en patrones propios de países desarrollados y se puede afirmar sin duda alguna que la transición está teniendo lugar de forma mucho más rápida que en Europa, (de hecho, se trata de uno de los procesos de transición más rápidos del mundo ${ }^{17}$ donde este mismo proceso tomó alrededor de un siglo y medio frente a medio siglo en el Magreb.

Sin embargo, también es cierto que hay que destacar que, precisamente, los patrones demográficos de estos tres países son bastante dispares. Si en el caso de Argelia asistiríamos según estas predicciones a un aumento muy importante de la población y en el caso de Marruecos, aunque significativo, sería notablemente inferior, en el caso de Túnez, es donde nos encontramos seguramente el modelo más cercano al de países desarrollados como en la mayor parte de la ribera norte del mediterráneo.

\subsection{Proporción de la población envejecida}

En cuanto a la proporción de población que nos podremos encontrar en el futuro, a tenor de las mismas predicciones de la ONU que hemos mencionado, se puede perfectamente ver cómo la proporción de población mayor de 65 años va a aumentar igualmente según va teniendo lugar esta transición demográfica: En Túnez para el año 2050, la población perteneciente a esta franja de edad debería encontrarse sobre los 2,5 millones (sobre un total de 12 millones) ${ }^{18}$, sobre los 8 millones (sobre un total de 46 millones) en Argelia ${ }^{19}$ y de 7

\footnotetext{
${ }^{9}$ United Nations (UN): "Algeria", World Population Prospects, Country Profiles, en http://esa.un.org/unpd/wpp/country-profiles/pdf/12.pdf.

${ }_{10}^{10}$ Population Pyramid: "Morocco 2010", en http://populationpyramid.net/Morocco/2010/.

${ }^{11}$ Population Pyramid: "Morocco 2050", en http://populationpyramid.net/Morocco/2050/.

${ }^{12}$ Population Pyramid: "Tunisia 2010", en http://populationpyramid.net/Tunisia/2010/.

${ }_{13}$ Population Pyramid: "Tunisia 2050", en http://populationpyramid.net/Tunisia/2050/.

14 Naciones Unidas (ONU): "Morocco", World Population Prospects, Country Profiles, en http://esa.un.org/unpd/wpp/country-profiles/pdf/504.pdf; United Nations (UN): "Tunisia", World Population Prospects, Country Profiles, en http://esa.un.org/unpd/wpp/country-profiles/pdf/788.pdf.

${ }^{15}$ Sandell, Rick: "La Demografía de nuestros vecinos: una preocupación geoestratégica", Fundación FAES, Cuadernos de Pensamiento Político (Enero/Marzo 2005), en http://www.fundacionfaes.org/record_file/filename/2999/CPP5_Demograf_adenuestrosvecinos_Sandell.pdf.

${ }^{16}$ Ben Bahram, Mehdi: " Le vieillissement démographique, un autre défi pour le Maghreb", Le Monde, 15 Mayo 2012, en http://www.lemonde.fr/idees/article/2012/05/15/le-vieillissement-demographique-un-autre-defi-pour-lemaghreb 1701045 3232.html.

${ }_{17}$ Abis, Sébastien (2012): Pour le future de la Mediterrannée : L'Agriculture, L'Harmattan, IREMO, p. 19.

${ }^{18}$ ONU: "Tunisia", op. cit..

${ }^{19}$ ONU: "Algeria", op. cit.
} 
millones finalmente en Marruecos (sobre un total de 39 millones) ${ }^{20}$. De estas cifras parece deducirse que el mayor envejecimiento se debería dar en Marruecos. Si bien, para el año 2050, los países del Magreb, Marruecos incluido, deberían encontrarse aún muy lejos de las escalofriantes proporciones de población mayor de 65 años que puede encontrarse en las estimaciones de los países más envejecidos del mundo como Alemania y Japón, con algo más de un tercio, no deja de ser éste un envejecimiento significativo.

Sin embargo, cabe acabar este párrafo mencionando la reflexión de Lutz, Samir y Scherbov en Lutz, Goujon, según quienes, se prevé que precisamente, la fecundidad siga bajando en los países de África y Oriente Medio, cosa que no sucede en Europa y que de mantenerse, podría suponer un desafío para estos países mayor que para los países de la ribera norte $^{21}$, si bien en lo que a regiones como el África Subsahariana se refiere, la población seguirá siendo joven.

\title{
1.4. El factor del éxodo rural como agravante
}

¿Qué consecuencias puede traer esto sobre la población rural cuyo envejecimiento es sin duda alguna una amenaza para la sostenibilidad de la producción agraria y por tanto para la seguridad alimentaria de estos tres países? Para ello, hay que tener en cuenta otros factores, que pueden tener unas consecuencias aún más graves para el equilibrio poblacional en el medio rural que la evolución demográfica en sí. En particular, estamos pensando en el envejecimiento de la población rural como resultado de un éxodo rural que drene al campo de su población más joven. En este sentido, podemos remitirnos a lo que dice la FAO a este respecto:

\begin{abstract}
"... ageing in rural communities usually manifests itself earlier and advances more rapidly than in the cities (Marcoux 1994, 2001; Skeldon 1999). By far the most important determinant is rural-to-urban migration which comprises mainly younger adults and thus increases the proportion of older persons 'left behind' in the villages...in the majority of poorer countries, ageing is predominantly a rural phenomenon and it is in the villages where the consequences of ageing are most felt (Stloukal 2001)... Rural outmigration, combined with falling fertility rates and increasing longevity, thereby increases the strain on older rural people, which can have negative consequences for their wellbeing as well as for their participation in rural development" ${ }^{22}$
\end{abstract}

El fenómeno de la migración rural ha sido en los últimos decenios un fenómeno muy destacado en los tres países del Magreb bajo estudio. Si en el año 1960 la proporción de población urbanizada se situaba en torno al $25 \%$ en el conjunto de los países del Magreb (Mauritania, Marruecos, Argelia, Túnez y Libia), en 1997 Marruecos y Argelia habían llegado al $50 \%$, mientras que Túnez se situaba ligeramente por encima con un $54 \%{ }^{23}$. Este proceso en nada se diferenciaba de aquellos por los que había transitado el mundo desarrollado en la

\footnotetext{
${ }^{20}$ ONU: "Morocco", op. cit.

${ }^{21}$ Lutz, Wolfgang y Goujon, Anne: "Capital humano, género y envejecimiento en el Mediterráneo. Perspectivas a medio y largo plazo", IEMed, Papers, $n^{\circ} 7$ (Febrero 2009), en http://www.iemed.org/observatori/areesdanalisi/arxius-adjunts/papersiemed/Paperiemed7.pdf.

${ }^{22}$ Stloukal, Libor: " Rural population ageing in developing countries: issues for consideration by FAO", FAO (Abril 2004), en http://www.fao.org/sd/dim_pe3/pe3 040401a1_en.htm.

${ }^{23}$ Lebrun, Léonce: "Le Maghreb", AFCAM (Abril 2010), en

http://www.afcam.org/index.php?option=com_content\&view=article\&id=188\&Itemid=124.
} 
ribera norte del Mediterráneo, de tal manera que nuestros tres países del Magreb se encuentran todavía a medio camino.

Tal y como las previsiones demográficas muestran, al Magreb aún le quedan por delante numerosos años de urbanización, siendo previsible igualmente que el mundo rural vaya bajando aún más en proporción poblacional. El peligro de un excesivo éxodo rural vuelve a resumirlo el PNUD mismo en su página web, en el apartado sobre Cambio Climático en el Magreb:

L'exode rural conjugué à l'urbanisation intense sur la côte méditerranéenne durant les dernières décennies ont augmenté la vulnérabilité des populations concernées tout en accentuant les facteurs qui contribuent aux changements climatiques $^{24}$.

Marruecos por ejemplo, puede resultarnos ilustrativo a este respecto si tenemos en cuenta las predicciones que se manejan: existen predicciones que sitúan a la franja de edad de menores de 15 años en una proporción de solo $17,7 \%$ a la altura del 2060, mientras que justo cien años atrás, en 1960, esta misma franja poblacional alcanzaba la cifra de 44,4\% sobre la población total $^{25}$. En cuanto a la población en el área rural de todo el mediterráneo, de los 41 millones en 1960 a 87 millones en 2010, se prevé una población rural de apenas 5 millones más, con 92 millones para el año 203026. Volviendo al ejemplo de Marruecos, nos encontramos de nuevo con que los datos demuestran cómo la transición de una población rural a otra urbana ha sido radical en los últimos decenios: de un $27 \%$ de población urbana en 1950, hemos pasado a cerca de un $60 \%$ en el año $2005^{27}$.

Un dato inquietante procede de la interpretación de los datos aportados por el CERED (Marruecos) por parte de la Investigadora Elisa Brey, que destaca que si bien se registra que solo algo más de un tercio del aumento de la demografía urbana en el período 1994-2004 procede de un saldo migratorio positivo desde el campo, esta inmigración bien podría ser más significativa como tendencia, pues se dio precisamente en los últimos años, aunque sí sea cierto que se enmarca en una disminución en comparación con fechas anteriores ${ }^{28}$. No hay que perder de vista que en términos generales, hasta el 2005, si bien la población urbana ha aumentado dos veces por encima de la población regional de África del Norte, y ello principalmente por el saldo migratorio positivo desde el campo a la ciudad, el hecho es que el aumento de la población nacional fue lo suficientemente importante como para que no hubiese despoblamiento del medio rural $^{29}$. ¿Será en cambio sostenible el medio rural si se mantienen tasas de éxodo rural significativas en el contexto de el crecimiento poblacional sensiblemente menor que se contempla en las previsiones vistas más abajo?

\footnotetext{
${ }^{24}$ ONU: " Le Maghreb, une zone clé face au changement climatique", Programa de Naciones Unidas para el Desarrollo (UNDP), en http://www.dz.undp.org/evenements/Chg Clim-magreb.html.

25 "Démographie marocaine: tendances passées et perspectives d'avenir", Centre des Études et Recherches Démographiques, Haut Commissariat au Plan (2005), en http://www.ondh.ma/Pdf_doc\%5CdemographieA4corrige.pdf.

${ }^{26}$ Abis, op. cit., p. 17.

${ }^{27}$ Brey, Elisa: "La Transición demográfica en Marruecos", Departamento de Ecología Humana y Población (Sociología II) Universidad Complutense de Madrid, en http://www.geps.es/uploads/tx_geps/DT002_III_2009.pdf, p. 14.

${ }^{29}$ Sandell, op. cit., p. 48.
} 
Una elevada cantidad de jóvenes emigrando a las ciudades y dejando detrás de sí a una población envejecida, siendo precisamente los magrebíes más jóvenes los que abandonen el campo con el fin de encontrar mejores oportunidades laborales, se traduciría por tanto en una merma tanto en términos cuantitativos como en términos cualitativos. Las consecuencias de un excesivo envejecimiento de la población agraria nos las ilustra de esta manera en su obra, Sebastien Abis:

\begin{abstract}
Le vieillissement des agriculteurs et la difficulté de transmettre aux nouvelles générations une exploitation sont des phénomènes qui progressent. En Tunisie, l'âge moyen des actifs agricoles est de 54 ans. Les jeunes peinent à s'investir dans ces métiers difficiles, souvent déconsidérés et parfois localisés dans des territoires marginalisés ${ }^{30}$.
\end{abstract}

Según datos de la Comisión Económica y Social para Asia Occidental (ESCWA en sus siglas inglesas) perteneciente a las Naciones Unidas, en Marruecos, la población rural pasará de estar por encima del 40\% a situarse cerca del $35 \%$ del 2010 al 2020, mientras que para esta misma fecha, siguiendo estas estimaciones, Argelia seguirá prácticamente los mismos porcentajes que su vecino occidental y Túnez seguirá estabilizándose a la baja por debajo del $10 \%$ de la población ${ }^{31}$. Este mismo informe, indica como en el período 19852015, el crecimiento de la población rural en sus segmentos poblacionales más activos (población joven) fue precisamente el menor ${ }^{32}$.

Otro dato inquietante, es precisamente el que ciertas predicciones contemplen que la población urbana por encima de los 65 años aumente más que la rural. Ello seguramente se vaya a dar como efecto de la falta de coberturas sanitarias para atender a este segmento de la población ${ }^{33}$, lo cual a su vez, sea un efecto del hecho de la decadencia económica del agro magrebí; de cumplirse estas predicciones, este éxodo de población mayor de 65 años será la fase final de un proceso cuyo primer eslabón, sea primero el éxodo de población joven hacia el medio urbano. Siguiendo con este mismo informe, se puede perfectamente constatar cómo son precisamente Marruecos y Túnez de los países del Mundo Árabe que tienen los índices de envejecimiento de la población rural más elevados, con un $18.5 \%$ y un $15.4 \%$ respectivamente, lo cual, según leemos en el mismo informe, se debe necesariamente a la emigración hacia el campo:

The old age dependency is set to increase as a result of individual ageing and gains in life expectancy for the 65+, coupled with migrations from rural to urban areas of working-age groups... ${ }^{34}$

\footnotetext{
${ }^{30}$ Abis, op. cit., p. 19.

${ }^{31}$ ONU: "The Demographic Profile of Arab Countries: Ageing of Rural Populations", Comisión Económica y Social para Asia Occidental (31 Diciembre 2007), en http://www.globalaging.org/ruralaging/world/2008/arabrural.pdf, p. 9.

${ }^{32}$ Ibid., p. 10 .

${ }^{33}$ Ibid., p. 12.

${ }^{34}$ Ibid., p. 13; hay que llamar la atención que en ese párrafo, el informe también menciona el posible retorno de población urbana mayores de 65 años hacia el medio rural, justo el fenómeno contrario que se mencionaba antes de emigración de este segmento de edad también; es difícil hacer predicciones fiables de este fenómeno.
} 


\subsection{Recapitulación}

Puesto que al contrario que Europa en su momento, El Magreb se enfrenta a este desafío de sostenibilidad agraria en un contexto particularmente crítico por darse el cambio climático, frente al cual no hicieron frente en su momento las naciones más modernizadas, y ello en una región particularmente vulnerable por su gran cercanía a la masa desértica del Sáhara, es razonable asumir que el margen para la caída en productividad del agro marroquí es mucho menor con respecto a países que no se ven amenazados por el fenómeno de la desertización, lo que exige por tanto reducir al máximo posible las debilidades estructurales de la agricultura magrebí.

Estos son los principales factores que incitan a la tan necesaria población joven a abandonar el medio rural. De esos factores estructurales y de las reformas necesarias para mitigarlos, pasamos a hablar en la sección siguiente.

\section{Factores que inciden en la baja sostenibilidad de la producción agraria en el Magreb y el envejecimiento de la población rural}

El envejecimiento de la población tiene precisamente una mayor incidencia en el colectivo cuya actividad laboral está vinculada a la producción agrícola. La tendencia hacia un fuerte envejecimiento de la población productora agrícola comenzó a manifestarse desde mediados de los años noventa, cobrando importancia por lo que acarrea tal fenómeno en cuestión de riesgos a la seguridad alimentaria de la zona.

Este envejecimiento de los agricultores no se debe solo a factores de cambios de fecundidad ni al aumento en la esperanza de vida, sino a múltiples variables y factores que confluyen en el fenómeno del éxodo rural y que pasamos a estudiar.

En este sentido podemos citar los datos aportados por el censo agrario del año 2001 de Marruecos donde se reflejaba que el $65 \%$ de los agricultores eran mayores de 50 años, el $37 \%$ contaba con más de 60 años y solo el 5,3\% eran mayores de 30 años. Por un lado encontramos que las propias explotaciones agrícolas adolecen de problemas estructurales. Un $70 \%$ de las explotaciones agrarias, de las cerca de 1,5 millones de explotaciones existentes son inferiores a las 5 has. y representan el $24 \%$ de la superficie agrícola útil. Dentro de este porcentaje, un 55\% tienen incluso una extensión inferior a las 3 has. Las explotaciones de 5 a 20 has., por su parte, constituyen más del $43 \%$ de la superficie agrícola útil ${ }^{35}$. Dado el reducido tamaño de las explotaciones, los explotadores se ven obligados a contar con varias propiedades de reducido tamaño que generan cada una de ellas ingresos frecuentemente escasos, siendo ello debido a que explotación de las mismas supone un sobreesfuerzo derivado de la no concentración parcelaria, no pudiéndose así maximizar el rendimiento e inversiones realizados en estas explotaciones. Esto tiene un efecto negativo pues se desalienta a las generaciones más jóvenes y no consideran como una salida profesional rentable la actividad agraria.

Túnez dispone de 5 millones de has. cultivables, siendo estas el 30,5\% de la superficie total del país. En cuanto a las explotaciones agrícolas es preciso apuntar que debido a la

\footnotetext{
${ }^{35}$ Oficina económica y comercial de España en Rabat (2010): Guía país Marruecos, en http://www.comercio.mityc.es/tmpDocsCanalPais/EEB361C4ACB64A8A6C6E7AEFA607B501.pdf.
} 
fragmentación constante a las que se ven sometidas las explotaciones agrícolas debido a los procesos de herencias familiares, más de la mitad de las explotaciones agrícolas cuentan con menos de 5 has., suponiendo este tipo de explotaciones un $9 \%$ de la totalidad de las tierras agrícolas tunecinas. Estas explotaciones son explotaciones de tipo familiar, las antiguas tierras comunales actualmente están siendo explotadas por las grandes empresas agrícolas. En Túnez existe una concentración de tierras bastante alta ya que más del $25 \%$ del total de las tierras cultivables están gestionadas por el 1 por ciento de los agricultores. Debido a esta situación desde 1980 la política de desarrollo rural llevada a cabo por las instituciones gubernamentales ha tratado de fomentar y proteger a las pequeñas explotaciones.

En el caso argelino la extensión media es de 4 has. lo cual es suficientemente adecuado para la producción rentable hortofrutícola o de cereal ${ }^{36}$. En el año 2010 se aprobó en consejo de ministros un nuevo proyecto de ley, que prevé la fijación del período de concesión de terrenos agrícolas para su explotación en 40 años, renovable y transmisible ${ }^{37}$.

A lo anteriormente mencionado hay que sumarle el hecho de que la mano de obra en la agricultura presenta una marcada variación interanual siendo un $81,27 \%$ de carácter permanente y un $18,73 \%$ estacional $^{38}$, dependiendo de la fase en la que se encuentre el producto. Esta estacionalidad incide en los agricultores más jóvenes como factor de desmotivación, al no ver éstos en esta actividad agraria una salida profesional ni viable ni estable.

Respecto a la composición de las explotaciones, los datos de la campaña 2005-2006 destacan que un $44 \%$ de los trabajadores son los propietarios de la explotación, un $21 \%$ coexplotadores, un $16,27 \%$ asalariados y un $18,73 \%$ jornaleros ${ }^{39}$. El subempleo es una práctica habitual en las comunidades rurales. Sin embargo, al tratarse de una actividad de economía sumergida su cuantificación resulta impracticable, siendo importante destacar que frecuentemente esta contratación está destinada a individuos con un actividad principal laboral, siendo la agraria bien complementaria, bien una ocupación laboral de transición hacia otros puestos de trabajo más atractivos.

Todo lo anteriormente señalado junto con el limitado nivel de formación técnica específica de los agricultores de estos países contribuye a que se mantengan tanto sistemas de cultivo ancestrales como un bajo nivel de tecnificación en las explotaciones agrarias, representando estos factores obstáculos obvios para la viabilidad de tales explotaciones a causa de la baja productividad, productividad por otra parte, que está parcial, pero directamente relacionada con la avanzada edad de los explotadores. Esta elevada edad condiciona las estrategias de gestión y explotación, ya que los agricultores de edad más joven manifiestan al contrario una mayor predisposición a una modernización y tecnificación que mejoren la productividad de las explotaciones. Los agricultores más jóvenes mantienen una visión a largo plazo que se traduce en su disposición no solo a contratar seguros que les permitan no solo recuperar su inversión ante un año de condiciones climáticas adversas, sino también a mejorar las explotaciones para que su productividad se beneficie de las mejoras. Los agricultores de edad más avanzada se decantan por el contrario por estrategias de autogestión basadas en el almacenamiento o el ahorro.

\footnotetext{
36 Oficina económica y comercial de España en Argel (2010): Guía país Argelia, en http://www.icex.es/icex/cma/contentTypes/common/records/mostrarDocumento/?doc=4425197.

${ }^{37}$ Ibid.

${ }^{38}$ Pro González, Maria José (2010): El caso de Argelia: El enfoque de la "subsistencia sostenible" y los seguros agrarios, CEIGRAM, Madrid.

${ }^{39}$ Ibid., p. 39.
} 
En cuanto a Argelia es preciso mencionar que ha venido presentando un claro déficit en materia de implementación de técnicas innovadoras en las explotaciones agrícolas, conservándose una tecnología anticuada, ya sea por la edad del dueño de la explotación y sus reticencias a la hora de hacer inversiones en tecnificación o debido al coste elevado de las mismas. En la actualidad, son empresas públicas las que proveen a nivel nacional de maquinaria, concentrándose en ellas un $60 \%$ de las ventas locales. Es en este aspecto de innovación y mecanización donde también incide el plan quinquenal 2009-2014 de renovación agrícola y rural.

En el caso de Marruecos nos encontramos con una situación en la que el índice de desempleo en las zonas rural asciende a tan solo un 4,30\% ${ }^{40}$, según los datos proporcionados por la dirección general de estadística marroquí en el año 2007 sobre población activa y desempleo. A priori, parece ser una salida profesional bastante atractiva, sin embargo hay factores que están favoreciendo que esta salida profesional no sea una opción atractiva para la población joven. Los factores más importantes son el cambio climático y la volatilidad de los precios agrícolas.

Otro factor que incide en el poco atractivo de la actividad agrícola son los precios. Aunque todos estos países han establecido unos precios de garantía para los productos básicos y los cultivos industriales y una amplia gama de subsidios para la compra de semillas maquinaria y fertilizantes, las quejas de los agricultores son bastante generalizadas sobre estos precios que permiten una mera supervivencia del agricultor a pesar de las ventajas que suponen en función de la notable fluctuación de las cosechas.

Esta fluctuación en el precio se produce de un modo más acusado en los cereales en función de las fluctuaciones en el nivel de precipitaciones y la especulación. En el caso de Marruecos estas fluctuaciones son acusadas. Su productividad sufre fluctuaciones directamente relacionadas con el nivel de precipitaciones cuya tendencia es decreciente y puede variar significativamente de un año a otro. Teniendo en cuenta que los cereales son los alimentos básicos de la dieta alimenticia de la población marroquí y que el sector de los cereales es el principal de la agricultura marroquí, podemos deducir la amenaza que esto supone para la seguridad alimentaria de Marruecos y el reto que representa para el desarrollo de la actividad agraria.

El Gobierno marroquí consciente de esta problemática ha puesto a punto en los últimos años un programa para modernizar y lograr una mayor diversificación de productos que permita que la producción agraria de este país no dependa en tanta medida de los cereales, fomentando en su lugar el cultivo de frutas y hortalizas así como hacer frente a las necesidades de una población en continuo crecimiento y proceso de urbanización. En relación a esto último el gobierno marroquí está tratando de mejorar la tasa de autoabastecimiento alimentario así como mantener un considerable flujo exportador en determinados productos (hortalizas y frutas) sobre todo con Europa.

La problemática que estos cultivos presentan es la falta de recursos hídricos por lo que el gobierno ha determinado que hasta el año 2030 construirá 50 pequeñas presas anuales que satisfagan las necesidades de agua de los agricultores. Este proyecto ha absorbido una parte importante de las inversiones públicas y el mayor porcentaje de las agrarias.

40 "Guía país Marruecos", op. cit. 
Los continuos esfuerzos por parte de las autoridades en materias como la extensión de la superficie de regadío, el equipamiento de los perímetros regados, las medidas de estímulo al uso de los factores de producción, la apertura al crédito agrícola, las labores de asesoramiento y capacitación agrícola de los agricultores y jóvenes rurales han tenido como consecuencia que la producción agraria de Marruecos haya sufrido un crecimiento aunque todavía insuficiente para garantizar el autoabastecimiento ya que el cambio climático y el proceso de desertización son factores no controlables por el gobierno y cuya repercusión en la productividad es directa.

Con este objetivo de aumentar la productividad del sector agrícola modernizando la agricultura, extendiendo la irrigación, investigación, el uso de la tecnología y las nuevas producciones más competitivas en los mercados internacionales el gobierno de Marruecos está desarrollando el Plan Marruecos Verde ${ }^{41}$.

Argelia por su parte, está desarrollando la Política de Renovación Agrícola y Rural, en el Plan Quinquenal 2010-2014 que incluye varios programas específicos como la modernización y difusión de los avances tecnológicos en las explotaciones agrícolas, con la ampliación de la superficie regada, el incremento de uso de fertilizantes, la mecanización, el uso de semillas seleccionadas, la introducción de un programa de concesión de créditos sin intereses (RFIG); la promoción de sistemas de arriendo financiero para el alquiler de maquinaria agrícola; la exención del IVA para la adquisición de insumos; la gestión de los riesgos asociados a la disminución del rendimiento agrícola y a los desastres naturales; la modernización del sistema de seguros, y el fortalecimiento de las organizaciones ${ }^{42}$.

En Túnez, con el objetivo de fomentar el desarrollo agrícola el gobierno ha tratado de mantener las rentas de los agricultores mediante una política de subvenciones estatales a los "inputs" agrarios tales como semillas, abonos, fitosanitarios o maquinarias, mejoras y ampliación de la superficie irrigada y primas a la inversión en agricultura Además, ofrece ventajas fiscales tales como la deducción de las sumas invertidas de los beneficios imponibles, la exoneración de los derechos de aduana, la suspensión del IVA y del impuesto al consumo debidos a la importación de equipos y tecnología productiva que no dispongan de un producto equivalente de fabricación nacional, la suspensión del IVA aplicado a los equipos fabricados localmente, y la exoneración del impuesto sobre los beneficios durante 10 años ${ }^{43}$.

Otro factor importante a mencionar es el previsible impacto del cambio climático y el incremento de la desertización y degradación de las tierras.

Con respecto al cambio climático, en los últimos años varios especialistas, incluyendo especialistas árabes, ha desarrollado o discutido diversos modelos de cambio climático sobre el Mediterráneo o el mundo árabe. Todos los modelos, con diversas variaciones, predicen un incremento de temperaturas importante, pudiendo alcanzar $\operatorname{los} 6^{\circ} \mathrm{C}$ a finales de este siglo. Los modelos del IPCC utilizados por diversos investigadores predicen un crecimiento de entre 1.2 $-1.5^{\circ} \mathrm{C}$ a $3.1-4.4^{\circ} \mathrm{C}$ para el año 2050 , dependiendo de los escenarios ${ }^{44}$. El US National

\footnotetext{
41 Cámara de Comercio de Marruecos en España: Oportunidades de comercio, en http://www.camarademarruecos.es/es/invertir-en-marruecos--Oportunidades-de-negocio.html.

42 "Présentation de la politique de RenouveauAgricole et Rural en Algérie et du programme quinquennal 20102014", en http://www.minagri.dz/pdf/Presentat\%20rar.pdf; FIDA: "Informe del $32^{\circ}$ período de sesiones del Consejo de Gobernadores Roma", 18 y 19 de febrero de 2009.

${ }^{43}$ Ministère de L'Agriculture et des Ressources Hydrauliques: XIème Plan de Developpement (2007-2011), Julio 2007; CIHEAM: "Perspectives des politiques agricoles en Afrique du Nord", Options Mediterranéennes, $\mathrm{n}^{\circ}$ 64, en www.wto.org/spanish/tratop_s/tpr_s/s152-4_s.doc.

${ }^{44}$ Véase una interesante discusión sobre escenarios y temperaturas en Marquina Antonio:"Prospects for Environmentally-Induced Migration from the Southern and Eastern Mediterranean to the European Union", en
} 
Intelligence Council, en un informe publicado en agosto de 2009 incluyó los modelos de varios estudios, llegando a predecir importantes caídas en las precipitaciones con una media de decrecimiento de precipitaciones anuales de un $12 \%$ para las zonas Mediterráneas y un 6\% para las zonas saharianas ${ }^{45}$.

Otros autores, de forma más optimista, proyectan una reducción de las precipitaciones de entre un 10 y un 20 por ciento a finales de este siglo. A lo que habrá que unir los cambios en las precipitaciones estacionales que afectarán seriamente a los cultivos agrícolas ${ }^{46}$. En la actualidad se constata que los países del Magreb central la precipitación media anual se está reduciendo.

La subida de temperaturas y caída de precipitaciones afectarán muy seriamente a la productividad de los cultivos agrícolas ${ }^{47} \mathrm{y}$, de no tomarse medidas, que se adivinan bastante costosas, inducirá una disminución de población dedicada a la agricultura, dados los rendimientos decrecientes, y un creciente envejecimiento de la población.

A lo que hay que añadir la desaparición de tierras cultivables debido al avance del desierto y a la degradación de la tierra. Buena parte del territorio de estos países es desierto. En Argelia el $90 \%$ de su territorio es desierto y en Túnez el 40\%. En Marruecos sólo el $22,1 \%$ del territorio es cultivable. De acuerdo con un informe de UNEP de 2006, en el norte de África, en los últimos cincuenta años, los sistemas tradicionales de cultivo y conservación se han deteriorado y la productividad ha disminuido en función de la creciente erosión del suelo debido al cultivo excesivo y al sobrepastoreo que ha reducido la fertilidad. Más del 57\% del total del territorio del norte de África está amenazado por la desertificación ${ }^{48}$.

El proceso de desertización es algo que preocupa en gran medida a los países del Magreb, en Argelia la lucha contra la desertificación se ha convertido en una de las principales preocupaciones del gobierno en relación al futuro de su agricultura, e integrada en la Política de Renovación Agrícola y Rural, en el Plan Quinquenal 2010-2014. En este sentido los programas de Renovación Rural se han centrado como una de sus cuatro prioridades en la lucha contra la desertización con la extensión y consolidación del barreras verdes, la protección y rehabilitación de las zonas de pastoreo y fijación de dunas, rehabilitación de las capas de plantas gramíneas alfa y desarrollo de la agricultura sahariana ${ }^{49}$.En el caso de Túnez la desertización avanza en el centro y el sur del país, la fuerte erosión a la que se ve sometida Túnez presenta síntomas de fertilidad decreciente en la mitad de los terrenos productivos. Las causas principales son la sobreexplotación de los recursos vegetales y unas prácticas agrícolas inadaptadas. Túnez ha puesto en práctica una política integrada de lucha contra la desertización que incluye una estrategia de lucha contra la erosión hídrica, una

Marquina, Antonio (ed.) (2011): Perspectives on Migration Flows in Asia and Europe, UNISCI/ASEF, pp. 239240.

${ }^{45}$ NIC (2009): North Africa: The Impact of Climate Change to 2030, en

http://www.dni.gov/files/documents/2009\%20Conference\%20Report_North\%20Africa_The\%20Impact\%20of\% 20Climate\%20Change\%20to\%202030.pdf, p. 3.

${ }^{46}$ Marquina, Antonio: "Prospects for Environmentally-Induced Migration from the Southern and Eastern Mediterranean to the European Union”, en Marquina, Antonio (ed.) (2011): Perspectives on Migration Flows in Asia and Europe, UNISCI/ASEF, p.238.

${ }^{47}$ Ibid., p.251.

${ }^{48}$ UNEP (2006): Africa environmental outlook 2, en http://www.unep.org/DEWA/Africa/docs/en/AEO2_Our_Environ_Our_Wealth.pdf.

${ }^{49}$ République Algérienne Démocratique et Populaire, Ministère de l'Agriculture et du Developpement Rural: Le Renouveau Rural .Evaluation de la mise en oeuvre, en http://www.minagri.dz/Reunions_des_Cadres/Reunion_des_cadres_06_01_2013/Renouveau_Rural.pdf. 
estrategia de fijación de dunas y una estrategia de desarrollo silvi-pastoral ${ }^{50}$ En Marruecos se han desarrollado políticas y programas para revertir la tendencia y atenuar la amplitud de la desertización, con políticas de construcción de presas y regadío, revalorización de tierras, planificación de perímetros de pastoreo y conservación y desarrollo de recursos forestales ${ }^{51}$.

Todos estos factores, tanto los climatológicos como los económicos, inciden de forma especial en la producción de cereales y juegan en contra de que la población joven opte por la agricultura como actividad laboral lucrativa, pues el nivel de incertidumbre al que se ven sometidos es muy elevado.

Por todo ello, la agricultura argelina, la marroquí y tunecina tienen actualmente el reto de mejorar la capacitación de sus agricultores, la introducción de técnicas agrícolas modernas, tomar medidas en torno al proceso de desertificación y maximizar los recursos hídricos a través de proyectos para la extensión del regadío, actualización de las estructuras catastrales obsoletas y optando por programas de concentración parcelaria que favorecieran la creación de explotaciones más grandes partiendo de la posesión de un número determinado de fincas pequeñas, mejorar la accesibilidad a la financiación de compras de semillas y plantas de alto rendimiento y maquinaria...etc., para intentar mejorar la baja productividad ya que todos los países del Magreb tienen rendimientos de los cereales muy por debajo del promedio de los países en desarrollo de $28.363 \mathrm{~kg} / \mathrm{ha}^{52}$, además de ser inferior a la media regional para el Cercano Oriente y África del Norte. La tasa más baja la encontramos en Marruecos la cual se sitúa entre el 30-70\% ${ }^{53}$. Los países del Magreb deben aumentar la productividad agrícola en términos de rendimiento por unidad de tierra y agua. A pesar del cambio climático, los procesos de desertización y la sobreexplotación del suelo, cabe un margen de mejora en la productividad agrícola de Argelia, Túnez y Marruecos si se realizan políticas de incremento en la superficie de regadío, evitando la sobreexplotación de los acuíferos, el uso de fertilizantes así como de Investigación, Desarrollo e innovación (I+D+i).

El crecimiento de la productividad en el sector agrícola juega un papel clave en el proceso de desarrollo de estos países, pues el sector agrícola es importante en su crecimiento económico y en la lucha para conseguir la reducción de la pobreza ${ }^{54}$. Pobreza que en las zonas rurales, donde se concentra la actividad agraria, es realmente alta.

Según el Banco Mundial, en el año 2009 la concentración de pobres en zonas rurales era la siguiente:

\footnotetext{
${ }^{50}$ République Tunisienne; Réalisations en matière de luttecontre la désertification, en http://www.environnement.nat.tn/sid/index.php?option=com_content\&task=view\&id=69\&Itemid=90.

${ }^{51}$ Haut Commissariataux Eaux et Forêts et à la LutteContre la Désertification: Luttecontre la désertification, en http://www.eauxetforets.gov.ma/fr/text.aspx?id=1020\&uid=39.

52 African Development Bank (AfDB) (2012): The Political Economy of Food Security in North Africa, en http://www.afdb.org/fileadmin/uploads/afdb/Documents/Publications/Economic\%20Brief\%20$\% 20$ The $\% 20$ Political\%20Economy\%20of\%20Food\%20Security\%20in\%20North\%20Africa.pdf.

${ }^{53}$ ICEX (2010): El mercado de la maquinaria agrícola en Marruecos.

${ }^{54}$ Christiaensen, L., Demery, L. and Kuhl, J.: "The (Evolving) Role of Agriculture in Poverty Reduction - An Empirical Perspective", Journal of Development Economics, vol. 96, no. 2 (2011), pp.239-254.
} 
Tabla 1. Población pobre (\%)

\begin{tabular}{|l|l|l|l|}
\hline País & $\begin{array}{l}\text { Porcentaje } \\
\text { población urbana } \\
\text { pobre }\end{array}$ & $\begin{array}{l}\text { Porcentaje } \\
\text { población } \\
\text { pobre }\end{array}$ & $\begin{array}{l}\text { Porcentaje de pobres en } \\
\text { zonas rurales }\end{array}$ \\
\hline Argelia & $10 \%$ & $15 \%$ & $52 \%$ \\
\hline Marruecos & $5 \%$ & $15 \%$ & $68 \%$ \\
\hline Túnez & $2 \%$ & $8 \%$ & $75 \%$ \\
\hline
\end{tabular}

Fuente: The World Bank (2009): Improving Food Security in Arab countries ${ }^{55}$

Estos índices de pobreza rural podrían verse reducidos si la productividad agrícola aumentara $\mathrm{y}$, a su vez, se produjera una mejora en los canales de distribución, los sistemas de almacenamiento y en las infraestructuras que facilitaran la accesibilidad a los mercados a los agricultores de las zonas rurales ${ }^{56}$. Según los datos del Banco Mundial el índice de las carreteras pavimentadas en Argelia en el año 2009 era un 74\%, sufriendo un incremento respecto al año anterior de tan solo un 0,5\%, el de Marruecos ascendió a un 70,3\%, siendo en el año 2008 el $62 \%$ de las carreteras la que se encontraban asfaltadas, en Túnez la cifra tanto en el año 2009 como en el 2008 se sitúo en un 75,2\% $\%^{57}$. Si bien las cifras nos pueden parecer lo suficientemente altas como para que el acceso a los mercados esté garantizado, hay que señalar que la mayoría de este porcentaje corresponde a carreteras ubicadas en las zonas urbanas y no rurales donde la actividad agraria se desarrolla.

Para todo ello los países del Magreb central tienen que aumentar el gasto público que vienen dedicando al sector agrícola ya que todos los factores que han sido mencionados en este apartado, de manera directa o indirecta, contribuyen al índice de rendimientos potenciales y por tanto, a que la actividad agraria sea vista por las nuevas generaciones de agricultores como una actividad lucrativa y una actividad alternativa a otras ocupaciones laborales. Como por ejemplo el sector secundario en el caso de Argelia y el terciario en el caso de Túnez, así como la zona costera de Marruecos; sectores por los que la mano de obra joven está viéndose atraída debido a la baja rentabilidad de la actividad agrícola en estos países, decidiéndose a abandonar el campo, favoreciendo el éxodo rural y poniendo por tanto en riesgo la seguridad alimentaria de estos países.

Junto a ello, hay que resaltar también la necesidad de diversificar las economías rurales y la oferta de servicios educativos y sanitarios que den un valor añadido a la permanencia en las explotaciones agrícolas familiares.

\footnotetext{
${ }^{55}$ The World Bank (2009): Improving food security in Arab countries, en http://siteresources.worldbank.org/INTMENA/Resources/FoodSecfinal.pdf, p.13.

56 "The Political Economy of Food Security...", p.19.

${ }^{57}$ Banco Mundial: Carreteras pavimentadas. (\% del total de carreteras) en http://datos.bancomundial.org/indicador/IS.ROD.PAVE.ZS/countries.
} 


\section{Conclusiones: la sostenibilidad del campo}

A modo de conclusión, a partir de lo que hemos podido ver en las páginas anteriores, podemos considerar que existen dos factores, o por lo menos dos grupos de factores relevantes en cuanto al potencial en productividad del campo magrebí. Uno de ellos, el que hemos visto en segundo lugar, corresponde a los problemas de parcelación agraria, a las dificultades para introducir mejoras técnicas y al mayor o menor apoyo de los poderes públicos para la introducción de todas las mejoras y reformas necesarias para que no solo se mantenga sino que se incremente la producción agraria. El segundo factor, y que es precisamente aquel con que iniciamos este capítulo, es el del fenómeno de envejecimiento de la población. ¿Por qué comenzamos precisamente con este factor? La razón es la siguiente: precisamente porque consideramos que será el capital humano que solo la población joven puede aportar el factor ineludible para que cualquier reforma que se lleve a cabo pueda llegar a buen puerto.

Aparte de eso, no hay que perder de vista que en el fondo, los dos factores que hemos mencionado se retroalimentan, pues las deficiencias estructurales de la agricultura magrebí impulsan a la población joven a emigrar al medio urbano, dejan el campo cada vez más en manos de una población envejecida, menos apta para una explotación efectiva y productiva. Ello inevitablemente dificulta la introducción de medidas de modernización y maximización, y por tanto reproduce y agrava el proceso. Envejecimiento y deficiencias estructurales son en este proceso de retroalimentación, tanto causa como efecto. Sin embargo, hay que tener en cuenta que el envejecimiento de la población rural que se prevé en el futuro va a tener lugar primordialmente por causa de la emigración de población joven y no tanto el envejecimiento demográfico, que, aunque enormemente rápido en comparación con la transición similar que está prácticamente finalizando en el mundo desarrollado, aún reserva significativos aumentos de población. Hay por tanto un aún considerable potencial de población joven.

Si no se realizan las inversiones oportunas, que son muy costosas, en el campo magrebí, esta emigración no podrá ser contenida, y aparte de acumular en las urbes masas de mano de obra joven que posiblemente no puedan absorber los sectores industriales y de servicios, privará al campo de la mano de obra necesaria para la sostenibilidad de la producción y de la seguridad alimentaria de los tres países del Magreb. Una vez que se hubiese consolidado esta inmigración, sería muy difícil revertir el proceso. 